\title{
LAS IDEAS DRAMATICAS DE PELLICER DE TOVAR
}

En 1635, enl la Academia de Madrid, Joseph Pellicer de Tovar, cronista de Castilla y Ireón, da a conocer su Idea de la Comedia de Castilla y Arte del estilo cómico. Se trata, a nuestro parecer, de uno de los más originales y sistemáticos tratados de teoría dramática con que cuenta el Siglo de Oro español. Este breve tratado de Pellicer, contenido en un manuscrito hasta hace poco inédito, había pasado prácticamente inadvertido ${ }^{1}$. Algo más conocido, pero nunca analizado rigurosamente, era otro breve ensayo de Pellicer que se publicó, en 1639 , con un título desorientador: Idea de la Comedia de Castilla deduzida de las obras cómicas del doctor Juan Pérez de Montalbán. Apareció en la fama póstuma que se dedicó a Pérez de Montalbán 2 . José Sánchez anota, de pasada, la semejanza entre ambos tratados y afirma que son «estudios distintos». El problema es curioso: no se trata de estudios distintos, sino de uno mismo usado dos veces con diferente propósito. Es decir, no hay ninguna diferencia, ni de fondo ni de forma, en la teoría dramática expuesta en ambos tratados. Después de leer y carear detenidamente los dos ensayos de Pellicer propcnemos la siguiente explicación. A raíz de la muerte de Pérez de Montalbán, en r638, Pellicer se vería forzado a colaborar, quizá con prisas, en su fama póstuma, que apareció en I639, y echó mano de su antiguo tratado, inédito, de 1635 . Lo que en I635 era un ensayo, de

1 Lo publica Jose SANChez en Academias Literarias del Siglo de Oro español, Madrid, 196r, pp. 82-89. Nosotros lo habíamos ya transcrito para la Preceptiva dramática española del Renacimiento y Barroco, en prensa, cuando vimos, con agradable sorpresa, el independiente hallazgo del señor Sánchez, aunque no añade comentarios explicativos y comete el error de fecharlo en I63I. En la portada del manuscrito aparece claramente la fecha de 1635 . Los textos citados en este trabajo proceden de la edición de Sánchez. Omitimos la paginación porque la comprobación es fácil, a causa de la brevedad del tratado. Son nuestros todos los subrayados que aparecen en los diversos pasajes reproducidos en este artículo.

2 Lágrimas Panegiricas a la temprana muerte del gran poeta Juan Pérez do Montalbán, Madrid, 1639. 
carácter general, sobre el teatro español (sin mencionar a Pérez de Montalbán, ni a ningún dramaturgo, salvo una sola vez a Lope de Vega) se "aplicó» ahora al teatro de Pérez de Montalbán. Aunque resultan muy forzadas las alusiones y elogios a Pérez de Montalbán (que es, en realidad, lo único nuevo en este segundo tratado de I639) eran teóricamente uposibles" por ser Montalbán fiel discípulo y seguidor de Lope de Vega, en cuyo arte se habia inspirado, en 1635 , Pellicer de Tovar al componer su original tratado $^{1}$. Por consiguiente, desde el punto de vista de la ideología dramática, el único documento sincero y genuino, sistemático y organizado, es el del discurso de 1635 , cuyos aspectos fundamentales vamos a estudiar.

Pellicer representa uno de los eslabones finales de la gran cadena de preceptiva dramática que inician, con contactos esporádicos, en el siglo xv, el marqués de Santillana y Juan de Mena ${ }^{2}$. El primer gran teórico que crea un clima nacional donde cien años después podrá surgir un Pellicer es Torres Naharro con su independiente y original clasificación de teatro «a fantasía" y "a noticia», esquema que se aparta de las tradicionales formulaciones aristotélicas ${ }^{3}$. Es importante también, en su definición de la comedia, un proceso de dignificación del género "comedia»: «... artificio ingenioso de notables y finalmente alegres acontecimientos, por personas disputado" *. Pellicer, intensificando este camino, llegaría al clímax de valoración de la comedia como el género literario «por excelencia». Pero hay que penetrar en el siglo XVII para encontrar dos importantes documentos literarios - de sobra conocidos por la crítica de nuestros díasen donde se respira una atmósfera relativamente próxima a Pellicer. Nos referimos al Ejemplar Poético (1606), de Juan de la Cueva y al Arte Nuevo de Hacer Comedias en este Tiempo (1609), de Lope de Vega. Por otra parte, en ambos tratados se dan curiosos paralelismos de circunstancias vitales que los diferencian considerablemente del tratado de Pellicer. Ambos ensayos son el producto de dos dramaturgos profesionales, en

1 Para ver las rivalidades, en otros terrenos, entre Pellicer y Lope, véase Damaso Alonso, Cómo contestó Pellicer a la befa de Lope, en Estudios y Ensayos Gongorinos, Madrid, 1960, 2.a edición, pp. 488-509.

2 MARQuís DE SANTILlana en su dedicatoria a La Comedieta de Ponça en Obras de Don Iñigo López de Mendoza, Marqués de Santillana, edic. de Josł AmaDOR DE LOS Ríos, Madrid, I852, pp. 93-95; y JUAN DE MENA, Coronación, Sevilla, $\mathrm{x}_{499 .}$.

3 Véase F. S. Escribano, Cuatro Contribuciones Españolas a la Preceptiva Dramática Murdial, Bulletin of the Comediantes, I961, XIII, pp. I-3.

- En Propalladia and other Works of Bartolomé de Torres Naharro, edic. de JOSEPH E. GIILIET, Brywn Mawr, 1943, vol. I, pp. 14I-143. 
donde la "experiencia" se sobrepone en intensidad (y no siempre se intercala en conexión lógica) a la especulación teórica. Además, en sus posturas, su aparente agresividad esconde un complejo defensivo de un arte perso nal cuyos alcances futuros no se calculan todavía con seguridad de triunfo. En otras palabras: hay mucho de experimento y defensa de un género nuevo, sentido paradójicamente con todos los perfiles de vigor, $\mathrm{y}$, al mismo tiempo, con las fluctuaciones vacilantes de un estilo no cristalizado. $\mathrm{Y}$ en la forma externa de sus exposiciones, también Juan de la Cueva y Lope de Vega ofrecen características similares, inexistentes en Pellicer: forma epistolar y redacción en verso. Lo epistolar produce un "tono" humano informal, en donde la amenidad y la viveza colorean las afirmaciones, que, por otra parte, pierden relieve de intelectual responsabilidad. A la forma poética, con su tiranía de la rima, acaso haya que achacar ciertas contradicciones o ligerezas. Pues bien, ninguna de estas características se dan en Pellicer, como veremos. Pero el ensayo de Lope de Vega, con su gran densidad teórica y original formulación, supone un verdadero puente tendido entre Cueva y Pellicer. $Y$ tiene, además, junto a las grandes diferencias ambientales y psicológicas con respecto de Pellicer, que acabamos de señalar, otras curiosas coincidencias. Los dos ensayos, el de Lope y Pellicer, emergen en un idéntico marco académico. Ambos surgen para cumplir con un compromiso oficial con la misma Academia, la Academia de Madrid. Y, coincidiendo en esto con Juan de la Cueva, se proponen estudiar solamente el teatro nuevo, en un momento y lugar muy concretos: la España contemporánea (leemos en los títulos: "Arte Nuevo de Hacer Comedias en este Tiempon, en el caso de Lope; "Idea de la Comedia en Castilla. Preceptos, del teatro de España y Arte del estilo moderno cómicon, por parte de Pellicer). En Lope de Vega lo importante es subrayar de una manera definitiva el valor de la tragicomedia, el público como constante inspiración y la evasión de definir la comedia (noye atento y del arte no disputesn), para resaltar su plenitud estética, indefinible y misteriosa 1. La actitud de Pellicer, teórico, será fundamèntalmente distinta al enfrentarse directamente, con rigor analítico, con el teatro nacional.

Entre los veinticuatro años de lapso que median entre El Arte Nuevo y la Idea de la Comedia se han levantado, como es sabido, muchas voces en favor y en contra del teatro nacional. Señalemos, de pasada, que la actitud global de los preceptistas en lenguas vulgar (por ejemplo Pin-

1 Para tener una idea de conjunto de la preceptiva dramática de Lope de Vega, véase LUIS C. PÉREZ y F. SÁnchez Escribano. Afirmaciones de Lope de Vega sobre Preceptiva dramática. Madrid, C.S.I.C., r96r. 
ciano, Cascales, González de Salas) es sorprendentemente negativa. La única excepción importante es la que representa Alfonso de Carvallo en su Cisne de Apolo. Poca influencia, por tanto, pudo recibir Pellicer de este grupo de preceptistas. Hay que llamar la atención sobre un importante tratado que consideramos el precedente inmediato de Pellicer. Nos referimos a la Invectiva de las comedias que prohibió Trajano y apologia por las nuestras que publicó Francisco Barreda en $1622{ }^{1}$. Citamos, a causa de su gran trascendencia, un largo párrafo de este tratado: -Advirtiendo primero, que las comedias que hoy gozamos dichosamente, son un orbe perfecto de la poesia, que encierra y ciñe en sí la diferencia de poemas, cuyas especies, aun repetidas, dieron lustre a los antiguos. Hay en las comedias nuestras la majestad, esplendor y grandeza del poema Epico. Tienen sus fábulas, sus episodios, y tal vez su verdad de historia, como el Epico. Hay también las flores y dulzuras sonoras del lírico, las veras y severidad del Trágico, las burlas y risas del cómico, los sainetes y salas del mímico, la gravedad y libertad de la sátira." Esta concepción del teatro nacional como el género de más grandeza y complejidad artística (afirmación audaz que en I609, cuando Lope escribió su Arte Nuevo, tal vez hubiese parecido exagerada irreverencia) pudo influir en Pellicer, cuando trece años más tarde, en I635, se planteó el estudio del teatro nacional no como un género incipiente y problemático, sino como una realidad aclimatada.

Con estos precedentes necesarios podemos ahora observar los más densos núcleos ideológicos del breve tratado de Pellicer de Tovar. La Idea de la Comedia de Castilla está presentada en forma de preceptos que alcanzan el número de veinte. Hay una breve introducción, muy importante como punto de partida. Alli, dentro del clima de afectada modestia, típico de semejantes ocasiones, se hacen dos afirmaciones fundamentales para comprender la génesis de este ensayo dramático. Sabemos que fue concebido y escrito en muy poco tiempo: uel que a mi me mando ocho dias ha que discurriese..... Mucho más trascendental es la segunda afirmación: «Seale, empero, disculpa a mi desconfianza el ser yo el primero que toma para este empeño la pluma, sin auer hasta oy, que yo haya visto, en nuestra lengua quien haya tratado por incidencia o de proposito esta materia.» Tras señalar la brevedad de tiempo consumido en su elaboración y destacar lo nuevo de su tema, pasa a subrayar su propósito y a elogiar sin reservas el género que se propone estudiar: "Es el intento, no

1 El mejor principe, Trajano Augusto: su filosofia, politica moral y económica deducida y traducida del panegirico de Plinio, ilustrado con imdgenes y discursos. Madrid, 1622, fols. 125r-141v. 
menos que dar preceptos a los mas eninentes hombres de esta Nación, en la accion mas admirable para las estrangeras que es la Comedia. Acaso no haya que atribuir completamente a exageración vanidosa el hecho de que Pellicer se arrogue, por así decirlo, el título de fundador de una nueva preceptiva dramática. Es verdad que, en sentido estricto, no había ningún cuerpo de doctrina teatral, con carácter normativo, sobre la comedia española. Ya expusimos nuestro punto de vista de que los casos de Juan de la Cueva y I,ope de Vega representaban más bien justificaciones personales $\mathrm{y}$ análisis incompletos del teatro contemporáneo, sentido como algo que se "está haciendo» todavía. También señalamos la despreocupación técnica de los preceptistas españoles en torno al teatro nacional, además de su postura intelectual pronunciadamente opuesta. Por otra parte, tampoco Pellicer podía manejar (y esconder su procedencia) fuentes extranjeras, ya que las otras naciones europeas se preocupaban más por la tragedia y los elogios a la comedia española, que el propio Pellicer destaca, tenían más un carácter admirativo que un análisis concreto de una técnica literaria. Nos hemos detenido algo en ver el alcance de la verdad en las afirmaciones de Pellicer, conocido su carácter jactancioso a través de un documentado artículo de Dámaso Alonso ${ }^{1}$. Aunque se podrían citar otros casos de honestidad y humildad científica por parte de Pellicer, como cuando escribe su ensayo sobre el poema épico y reconoce de antemano que hay muy poco de original y que se basa solamente en ajena información ${ }^{2}$.

Esta breve introducción de Pellicer termina con un intenso panegírico: «Yo confieso que la comedia, como está oy, es el poema más arduo para intentarlo, i más glorioso para conseguido que tienen los Ingeniosı. Esta afirmación es audaz y revolucionaria. Supone un viraje rotundo de los valores literarios. La antigua concepción aristotélica de los dos grandes géneros, tragedia y épica, había sufrido un significativo cambio de factores en el Renacimiento: épica y tragedia (excepto en Castelvetro que sigue fiel a Aristóteles en anteponer la tragedia a la épica) ${ }^{3}$. Pellicer, ahora, considera la comedia el género más difícil y

1. DÁmaso Aronso, Todos contra Pellicer, en Estudios y Ensayos Gongorinos, Madrid, $1960,2 .^{\mathrm{B}}$ edic., pp. $462-487$.

2 En Epilogo de los preceptos del poema heroico, Madrid, 1635, publicado por Još SÁnchez, op. cit., pp. 89-93. Alli Pellicer confiesa: "que no lleva mas de mio [este tratado] que haverle copiado de otros maestros, riesgo que padece siempre quien escribe por donde otros pautaron".

3 Estas viejas cuestiones se han tratado de nuevo, con precisión, en AUGUST Buck. Italienische Dichtungslehren. Von Mittelalter bis zum Ausgang der Renaissance. Tübingen. r952. 
glorioso, excluyendo los tradicionales géneros, pese al lastre autoritario de la voz de Aristóteles. Recordemos que habíamos registrado una opinión parecida del licenciado Barreda, que pudo influir en Pellicer. Nos hemos detenido especialmente en la introducción, porque es donde, históricamente, se perfila más recortadamente la gran trascendencia teórica de su personal y vigorosa silueta crítica.

Es sintomático que, en su primer precepto, se plantee Pellicer el problema moral, es decir, la justificación moral de un género literario: «Porque començando en su primer precepto, que es el fin que debe tener la comedia, se constituye voluntariamente el que la hace por maestro publico del pueblo que le esta oyendo, de cuyos avisos depende la enseñança de todo el concurso. Sin duda que en este momento todavía están presentes en la memoria, de alguna manera, las controversias acerca de la licitud del teatro y sus representaciones ${ }^{1}$. Ni Juan de la Cueva ni Lope de Vega, aunque también hablaron de la finalidad educativa del teatro y arte en general -especialmente Juan de la Cueva-, se vieron precisados, antes de iniciar sus exposiciones, con una toma de contacto con el problema moral. Pinciano y Carvallo comenzaron sus disquisiciones sobre el teatro con una postura más aséptica y filológica: discuten la etimologla de la palabra comedia. Claro está que más adelante ambos se ocupan del problema moral, sobre todo Carvallo. Pellicer, por el contrario, omite el tema etimológico, hecho que corrobora, de pasada, nuestra impresión de que no se sirvió de las famosas poéticas nacionales para su tratado.

En este mismo precepto creemos que ofrece gran interés una, acaso, novedosa conexión con la doctrina platónica del furor divino, al afirmar que el poeta debe ensalzar las virtudes morales, de modo "que despierte con furor divino en los oyentes un fervor activo de imitar aquello que miral. En esta mención al furor divino se inyectan tres aspectos dignos de consideración. En primer lugar, se aplica a la comedia un principio tradicionalmente empleado para justificar y dignificar la profesión poética; ni Juan de la Cueva, ni Lope de Vega, ni los preceptistas españoles mencionan el furor divino al estudiar el teatro nacional. Por otra parte, el furor divino siempre se asociaba al tema de la inspiración, como ya habían hecho Petrarca y Boccaccio, destacando sus raíces subjetivas; ahora, con Pellicer, este concepto se objetiviza para ver su influencia externa en el espectador. Por último, notamos que esta influencia no se analiza como una inflamación, por contagio de placer estético, sino como una penetra-

1 Véase Emmio Cotarelo y MORI, Controversias sobre la licitud del teatro, Madrid, r904. 
ción capaz de provocar una imitación activa en la buena conducta de los hombres. Fn resumen, la doctrina tradicional del furor divino ha sufrido dos desplazamientos: $a$ ) su aplicación al teatro; $b$ ) sus repercusiones morales o didácticas.

Este primer precepto de Pellicer es de los más extensos y se cierra con una definición de la comedia en un plano estrictamente moral: "es una accion que guie a imitar lo bueno y a escusar lo malon. Aquí vemos un eco de la antigua definición atribuida a Cicerón por Donato: «imitatio vitae, speculum consuetudinis, imago veritatisn. Ya popular en la Edad Media, esta definición pasó a las Poéticas y subsistió durante todo el Siglo de Oro. Lo significativo es que en Pellicer, de los tres miembros de la definición, sólo subsista el elemento más estricta y uprácticamente moral, «espejo de costumbres», es decir, "de buenas costumbres" en Pellicer. Esta preocupación moral llega a manifestarse en detalles concretos, como en el precepto quinto. En este precepto señala que, en las comedias que tratan de amores, los protagonistas deben ser personas libres ii no atadas al yugo santo del matrimonion. $Y$ una preocupación ejemplificadora tiñe, seguramente, el precepto decimoquinto, que «es pintar el heroe i la heroida mas perfectos en meritos personales que a los demas, comprehendiendo en la ventaja a los reyes\%. Por supuesto que esta doctrina es muy aristotélica.

Tras resolver un problema básico como el moral, presupuesto necesario en la sociedad española de la época para la existencia de un género literario, Pellicer aborda otro núcleo teórico de gran alcance: el estilo. Es sabido que en el Siglo de Oro, por lo general, estilo equivale a género. La otra acepción frecuente es modo, con sus habituales connotaciones literarias de bajo o elevado, es decir, estilo bajo o elevado, con el que se incide de nuevo en los géneros literarios: algunos gloriosos por sus temas, y otros humildes por lo tratado y la forma de presentación. En otras palabras, al hablar de estilo más que de recursos expresivos individuales en un escritor, estamos ante el dilema de seleccionar un vehículo literario muy determinado: tragedia, comedia, épica y lírica. Pellicer nos habla de comedia, equivalente a teatro español. Desde Cascales, en sus Tablas Poéticas, la división tragedia y comedia quedó fundida en un nuevo concepto: drama; aunque sólo a efectos de simplificación terminológica; así los cuatro géneros aristotélicos se reducían a tres ${ }^{1}$. Pero la comedia quedaba muy mal parada, y la mezcla de tragicomedia, considerada monstruosa. De aquí que Pellicer, dieciocho años más tarde, tiene todavía que

1 Véase Margarete Newels. Die dramatischen Gattungen in den Poetiken des Siglo de Oro. Wiesbaden I959, p. 9. 
luchar una batalla teórica, pero con la ventaja de una práctica de teaíro nacional triunfante, que la hacia mucho más fácil. Pero en realidad, Pellicer no libra ninguna batalla concreta por lo arriesgado que serfa, de todos modos, enfrentarse directamente con los partidarios de un estilo o género determinado. Pellicer utiliza una ingeniosa estrategia: adopta una actitud ecléctica y englobadora. Leemos en el segundo precepto: "Y $Y$ aunque en mi opinión la Comedia no lo tiene determinado [estilo], hallo en esto mayor excelencia, por ser un compendio de los tres estilos, tragico, lyrico y heroyco. En el precepto decimosexto presenta otra vez su visión del teatro como un género sintético y armonizador de todas las tendencias literarias. Alli señala tres tipos de comedias. A través de los textos que vamos a citar se observará que su pretendida clasificación queda debilitada por las contaminaciones que Pellicer propugna. $O$, dicho de otra manera, el teatro nacional queda interpretado como una materia neutra, de gran capacidad aglutinante, en la que se confunden todos los géneros. Nos habla de la comedia heroica, que, para agradar al público, puede adornarse «de episodios líricos y trágicos». Después dice que la comedia lirica "que se funda en lo melancolico que contiene la maraña amorosa d dulce, tiene que tener elementos tragicos y heroicos." Por fin, nos habla de la comedia trágica "que se funda en lo melancolico y funebre de la lastima, pero que debe tambien tener elementos liricos y heroicos.n La moderna ciencia de la literatura, después de más de tres siglos, coincidirá con Pellicer al afirmar que la obra literaria perfecta debe ser una conjunción de los tres géneros tradicionales 1.

Al plantearse el problema del estilo, en el segundo precepto, también se plantea Pellicer un problema técnico importante, a saber, la métrica. Pellicer está detrás de una tradición conocida: Argote de Molina, Juan de la Cueva y Lope de Vega, además de los preceptistas en lengua vulgar. Pero Pellicer no se sujeta a ningún esquema preestablecido y expone sus preferencias respecto a metros adaptables a unas situaciones dramáticas. Y aquí actúa una vez más con independiente personalidad. Para la comedia trágica recomienda los tercetos y también las octavas, canciones, silvas y romances. Para el estilo lírico que, a menudo, aparece en la comedia, aconseja décimas, endechas, liras, quebrados, glosas, sonetos y romances. Para la comedia de estilo heroico acepta (obsérvese que coincide con la comedia de estilo trágico) octavas, canciones, silvas y romances. Los romances son aptos, por consiguiente, para todos los

I Asi, por ejemplo, EmIr StaIGer, en Grunbegriffe der Poetik, Zürich, 195x, 2. edición, p. ro, afirma: ajede echte Dichtung an allen Gattungsideen in verschiedenen Graden und Weiser beteiligt ist. 
estilos. Más adelante afirma que la comedia puede usar en general de coplas de arte mayor, pero que las redondillas o quintillas sólo deben servir "para enlaçar la maraña de la Comedia.» Tenemos, pues, que, en la métrica, se nota también, aparte de su independencia de juicio, su preocupación de considerar la comedia española como un género ecléctico, en el que la métrica puede ayudar a señalar contornos y matices. Mas, a la postre, los esquemas métricos son intercambiables y arrollados por el poder englobador del género dramático.

Pellicer, en el precepto onceno, ensaya una sencilla tipología de la producción dramática española, porque, aunque todo el teatro español es conocido con el nombre genérico de comedia, comedia, propiamente hablando, es sólo tla que consta de caso que acontece entre particulares donde no ai principe absoluto." Aquí su pensamiento es tradicionalmente aristotélico y no se ajusta a la realidad de lo observado por el propio Pellicer. Pero, al señalar los otros tipos posibles, se expresa con personal vivacidad en dos de ellos: la fábula y la tragicomedia. Para Pellicer la comedia de tramoya es fábula y su actitud negativa la consignó en el precepto noveno. Para la tragicomedia emplea una definición que lleva un cuño muy personal: «aquella donde se muere el heroe que es el primer galán, es tragi-comedian 1. Para la tragedia sigue, lo mismo que para la comedia, el esquema tradicional aristotélico «aquella donde se introduce el rey es tragedia.» En este precepto, que adolece de elaboración insuficiente, junto a la doctrina tradicional, recogida con cierto apresuramiento, vemos que asoman destellos personales, como es característico de todo el tratado que estamos revisando.

En su afán de aprovechar las teorías poéticas de carácter general en una nueva vitalización del género dramático, tal como había hecho con el furor divino, usa el argumento de poesía (en el caso de Pellicer, poesía dramática), como ciencia universal, dentro de la topología (panegíricos de la poesía. Este precepto, que es el duodécimo de su tratado, sería un ejemplo más que podríamos añadir al (topo» que determinó Curtius ${ }^{2}$. $\mathrm{Y}$ además, puesto que Pellicer siempre tiene presente el conflicto de los géneros, empieza por «acercar» el drama a la poesía épica y por insistir en el carácter aglutinante del teatro español: «que si para hacer versos, no más, es necesaria la inteligencia de las ciencias, para hacerlos atados

1 Curiosa definición que acaso arrojase alguna luz en el difícil problema que estudió EDWIN S. MORBY. Some observations on Tragedia and Tragicomedia in Lope. Hispanic Review, 1943, XI, pp. 185-209.

2 Véase ERNST ROBERT CURTrus. Europäische Literatur und lateinisches Mittelalter. Bern, 2. ${ }^{\text {a }}$ edic. I954, p. 530 y s. 
a leyes de raçon cassi epica, forjada de todos los estilos, porque no ha de ser precisa». Después, enumera los diversos conocimientos necesarios. E1 poeta que escriba autos sacramentales o comedias devotas precisará la teología. Para sutilizar sobre el amor, la Filosofía moral. Y para casos legales, la jurisprudencia; para aplicación de remedios, la medicina. Para hablar de la guerra, necesitará la matemática y la perspectiva. Y así Pellicer sigue enumerando diversas ciencias como la astrología, la historia, la geografía. Pero, además, el poeta dramático debe ser gran cortesano y usaber las agilidades y juegos de pelota, esgrima, axedrez, naipes, tablas y dados». Sin olvidar la marinería, la cetrería y hasta las artes mecánicas.

Pero claro está que, para muchos aspectos doctrinales que atañan al «arte» en general, Pellicer utiliza un material tradicional. Así, en lo relativo al decoro y verosimilitud ${ }^{1}$, sigue de cerca las teorías de Aristóteles y Horacio, pero siempre vivificadas con expresiones pintorescas. Este material queda revisado por Pellicer, especialmente en los preceptos tercero, cuarto y decimoctavo. Anotemos algunas plasmaciones peculiares de la idiosincrasia lingüística de Pellicer. Leemos, en el precepto tercero, que el poeta debe alternar la materia que trata siendo «ya tierno, ya hinchado, ya humilde, ya soberbio, ya con mesura, ya con despejo, ya severo; ya apacible, siendo cameleon de efectos contrarios...» En el precepto cuarto se nos dice que el poeta debe revestirse de los mismos afectos que escribe para que parezcan verosímiles y que umientras no sacare a los semblantes que le escuchan aquellos contrariedades de que se compone la escena o contiene floxedad lo escrito, o tibieça lo representado."

En el precepto dieciocho aparece una pintoresca ejemplificación del problema del decoro, al afirmar que se deben uescusar las acciones indecentes de los personajes graves, como son comer en las tablas, desnudarse, cantar i otras, que solo son para la graciosidad».

Otras veces Pellicer repite puntos (sin apenas nada nuevo en el enfoque) que se han ido formando con la teoría y la práctica del teatro nacional. Así, por ejemplo, en el precepto sexto, donde recomienda los celos, recurso cotidiano de nuestras tablas. En el precepto octavo, "que es la elecion del caso, ya sea historico, ya apocrifo", estamos ante un calco evidente de la clasificación de Torres Naharro de "comedia a noticia" y "comedia a fantasía». E1 precepto decimotercero "es procurar no dexar

1 Para comprender el alcance de la «verosimilitud» en España, véase ALBERTo Porgueras Mayo. El Problema de la Verdad Poética en el Siglo de Oro, Madrid. Ateneo, 1961. 
nunca solo el tablado que será mucha gala del discurrir»; precepto parecido propugnó Lope de Vega en su Arte Nuevo: "Quede muy pocas veces el teatro sin persona que hable [... $]^{1}$. En el precepto catorce propone el término de "jornadas" en vez de "actos", neologismo que Pellicer pudo aprender en su inventor Torres Naharro, o en sus seguidores Juan de la Cueva y Lope de Vega. Pero Pellicer extrema aquí su rigor hasta la pedantería: «Cada jornada debe constar de tres scenas, que vulgarmente se dicen salidas, i a cada scena le doy trescientos versos, que novecientos es suficiente circulo para cada jornada [...]». Para el precepto diecinueve, en el que recomienda "escusar aquella familiar llaneça que vemos cada dia entre el señor y el criado, fiandole los mas importantes secretos", pudo tener en cuenta críticas semejantes, sobre todo la de Cristóbal Suárez de Figueroa en su Pasagero: "graceja el lacayo con el Señor, teniendo por donaire la desvergüenza» ${ }^{2}$. En cuanto al último precepto, el número veinte, que recomienda "escrivir primero la traça o maraña de la comedia en prosa», lo aprendió en el Arte Nuevo: "el sujeto elegido escriba en prosa.» Aunque no consta que Lope de Vega lo practicase nunca ${ }^{3}$.

En todo este cuerpo de doctrina, desarrollado con tanta sagacidad y expuesto con tanta sistematización lógica, no podía faltar algún consejo absurdo y retrógrado, como el décimo, que trata del término de la acción "que yo le alargo a veinte $i$ quatro horas, solamente i es sobrada la distancia para cualquier suceso». Aquí, inexplicablemente, Pellicer sigue a los férreos aristotélicos italianos, que fueron más aristotélicos que el propio Aristóteles que, como es sabido, jamás propugnó la unidad de tiempo. Lope de Vega fue todavía más liberal que Aristóteles en su Arte Nuevo y en la práctica. Pero Pellicer parece echarse la manta a la cabeza y olvidarse de todo esto.

Otro aspecto curioso del tratado de Pellicer, imposible de estudiar aquí, es su terminología literaria que aportaría interesantes connotaciones para el día que se estudie la terminología dramática de nuestro Siglo de Oro. Aludimos a vocablos como «traça o maraña», "maraña o contexto», "gracejo», "novela», etc. Históricamente interesante, al final de su tratado, es la respetuosa alusión a don Luis de Góngora en su fracaso como dramaturgo.

1 Para las referencias al Arte Nuevo de Lope seguimos la edición de A. MORELFatro, en Bulletin Hispanique, III, pp. 364-405.

2 CRISTÓbAl SUÁREZ DE FigUEROA. El Pasagero, advertencias utilísimas a la vida humana. Madrid. Renacimiento, 1913, p. 75.

3 Véase Ménéndez Peidayo. Historia de las Ideas Estéticas en España. $3 \cdot^{\mathrm{a}}$ edicićn. Madrid, C. S. I. C. 1962, p. 298. 
Insistamos para terminar _en esta primera toma de contacto con las ideas dramáticas de Pellicer - que nuestro autor opera con un material preceptivo tradicional que se respiraba en el ambiente, sobre todo a través de la teoría y la práctica de Lope de Vega. Pero lo que hemos tratado de puntualizar es su esfuerzo constante por encontrar nuevos puntos de vista y expresarlos con personal originalidad. $\mathrm{Y}$ lo más destacado de este tratado, que, para nosotros es uno de los más importantes del Siglo de Oro, es la perspectiva histórica que se vislumbra ya en la fecha que fue escrito. Ya era posible dogmatizar y elogiar sin reservas. Esto posibilita también la construcción de una teoría detallada, sistemática y completa, de validez general.

\author{
A. Porqueras mayo \\ (Universidad de Missouri) \\ F. SÁNCHez Escribano \\ (Universidad del Colorado)
}

